Association for Information Systems AIS Electronic Library (AISeL)

Wirtschaftsinformatik Proceedings 2005

Wirtschaftsinformatik

February 2005

\title{
Market Engineering am Beispiel elektronischer Immobilienmärkte
}

Andrea Krause

Universität Karlsruhe (TH)

Matthias Kunzelmann

Universität Karlsruhe (TH)

Follow this and additional works at: http://aisel.aisnet.org/wi2005

\section{Recommended Citation}

Krause, Andrea and Kunzelmann, Matthias, "Market Engineering am Beispiel elektronischer Immobilienmärkte" (2005). Wirtschaftsinformatik Proceedings 2005. 14.

http://aisel.aisnet.org/wi2005/14

This material is brought to you by the Wirtschaftsinformatik at AIS Electronic Library (AISeL). It has been accepted for inclusion in Wirtschaftsinformatik Proceedings 2005 by an authorized administrator of AIS Electronic Library (AISeL). For more information, please contact elibrary@aisnet.org. 
In: Ferstl, Otto K, u.a. (Hg) 2005. Wirtschaftsinformatik 2005: eEconomy, eGovernment, eSociety; 7. Internationale Tagung Wirtschaftsinformatik 2005. Heidelberg: Physica-Verlag

ISBN: 3-7908-1574-8

(C) Physica-Verlag Heidelberg 2005 


\title{
Market Engineering am Beispiel elektronischer Immobilienmärkte
}

\author{
Andrea Krause, Matthias Kunzelmann \\ Universität Karlsruhe (TH)
}

\begin{abstract}
Zusammenfassung: Im Gegensatz zu online Job-oder Automobilbörsen leidet der internetbasierte Immobilienmarkt unter einem hohen Maß an Fragmentierung. Trotz der einsetzenden Konsolidierung konnte aufgrund von marktimmanenten Problemen bisher keine der existierenden Börsen eine kritische Masse erreichen. Vor diesem Hintergrund und in Anbetracht der Tatsache, dass sich die Erosion von Kleinanzeigen aus den Printmedien hin ins Internet fortsetzen wird, wurde eine Case Study durchgeführt, die das Design und die Erfolgsfaktoren von internetbasierten Immobilienbörsen analysiert.

Aufbauend auf diesen Ergebnissen wird im vorliegenden Beitrag unter Nutzung des Market-Engineering-Prozesses eine elektronische Immobilienbörse gestaltet, die aufgrund ihrer Marktstruktur, der Integration von gewerblichen und privaten Anzeigen sowie der angebotenen Services das Potenzial besitzt, die kritische Masse zu überschreiten. Dies würde neben der Nutzung von Netzeffekten und der Realisierung von Economies of Scale die Konsolidierung unter den Immobilienbörsen vorantreiben.
\end{abstract}

Schlüsselworte: Market Engineering, Immobilien, Case Study, Market Design, Internetökonomie, e-Business

\section{Motivation}

Durch seine zunehmende Verbreitung hat das Internet in vielen Bereichen des täglichen Lebens stark an Bedeutung gewonnen. Nach der anfänglichen Hypephase und der darauf folgenden Ernüchterung wurden Begriffe wie e-Business oder eCommerce nach und nach systematisch mit Inhalten gefüllt und tragfähige Geschäftsmodelle, für die neue Art zu wirtschaften, entwickelt. Aktuell haben sich sowohl zwischen Unternehmen und privaten Haushalten (B2C) als auch zwischen Unternehmen (B2B) über elektronische Medien abgewickelte ökonomische Beziehungen etabliert [Zerd 01, S. 219]. Durch das Aufkommen von online Auktionen wie z. B. eBay wurden schrittweise auch private Haushalte in die Lage ver- 
setzt, untereinander internetbasiert und strukturiert nach festen Regeln ${ }^{1}$ rechtskräftige Geschäfte zu tätigen.

Neben Angeboten wie eBay lässt sich auch an domänenspezifischen OnlineMärkten, beispielsweise im Automobilsektor oder dem Arbeitsmarkt, ein Trend weg vom Print- und hin zum elektronischen Medium erkennen [Frey ${ }^{+} 03$, S. 6f]. Dass sich die Anbahnung und z. T. auch der Abschluss von Geschäften über das Internet mit und zwischen Endkunden in einigen Bereichen bevorzugt etabliert hat, ist auch auf den Datencharakter der entsprechenden Produkte zurückzuführen. Dieser ermöglicht eine schematische, strukturierte Erfassung der Objektattribute und ist daher datenbanktechnisch umsetzbar. So lassen sich bestimmte Anzeigen des Rubrikenmarktes wie beispielsweise Pkws, Stellenangebote oder auch Immobilien jeweils durch eine einheitliche Struktur beschreiben [Zerd03, S. 2]. Diese Strukturierung erfolgt anhand der jeweiligen Objektattribute, die es den Benutzern nach ihrer Erfassung in einer Datenbank erlauben, aus den vorhandenen Objekten durch Spezifikation einer Anfrage die Objekte zu selektieren, die den eigenen Anforderungen am Besten entsprechen. Dieser Suchkomfort und die hohe Qualität der Treffer gegenüber einer Suche im Printmedium Kleinanzeigenblatt sowie die erweiterten visuellen Darstellungsmöglichkeiten, die ständige Verfügbarkeit und hohe Aktualität der Angebote haben zum nachhaltigen Erfolg dieser OnlineMarktplätze geführt [Zerd03, S. 2].

Während im Bereich der Online-Kfz-Börsen und der Stellenmärkte eine schnelle Konsolidierung stattfand, aus der wenige große Anbieter hervorgingen, leidet der Online-Immobilienmarkt weiterhin unter einer gewissen Fragmentierung [RoBö01, S. 107]. Obwohl die räumliche Zersplitterung des Immobilienmarktes als immanent angesehen werden kann und auf die Marktteilnehmer nicht störend wirkt, stellt die weitere Fragmentierung der Teilmärkte in diverse OnlineAngebote, Printmedien und Maklerverbände ein erhebliches Problem dar [Jenk96, S. 25ff]. Um die aktuelle Struktur des Immobilienmarktes in Deutschland und die Anforderungen der Handelsteilnehmer an den Markt zu erfassen, wurde in $\mathrm{Zu}-$ sammenarbeit mit der Quoka $\mathrm{AG}^{2}$ eine Fallstudie durchgeführt. Die Erkenntnisse aus der Fallstudie wurden genutzt, um einen zukunftsweisenden OnlineImmobilienmarkt ${ }^{3}$ zu konzipieren.

Die Gestaltung des Immobilienmarktes erfolgte anhand der ersten vier Phasen des Market-Engineering-Prozesses von [Wein ${ }^{+} 03$, S. 639]. Das Ziel der Studie ist es,

$1 \quad$ In der Literatur wird die Menge aller Regeln wie beispielsweise bei [Neum04, S. 19] unter dem Begriff Institution zusammengefasst.

2 Mit 250.000 Kleinanzeigen pro Woche und davon 6.000 in der Wohnimmobilienrubrik ist die Quoka AG der Marktführer in Süddeutschland im Bereich gedruckter Kleinanzeigen.

3 Die Begriffe online, elektronisch und internetbasiert werden ebenso wie die Begriffe Immobilienbörse, Immobilienmarkt und Immobilienmarktplatz aus sprachlichen Gründen als inhaltlich gleichberechtigt nebeneinander verwendet. 
einen Online-Immobilienmarkt zu konzipieren, der durch umfassende Objektbeschreibungen, innovative Funktionalität und zusätzliche Services für die Teilnehmer einen höheren Nutzen als bestehende Immobilienbörsen stiftet und somit das Potenzial besitzt, zu einem konsolidierten (Teil-)Markt zu avancieren. Durch die große Anzahl der bereits in den Printmedien der Quoka AG vorhandenen Immobilienanzeigen sowie die umfassende konzeptionelle Berücksichtigung von Makleranzeigen besteht Grund zu der Annahme, dass nach der Umsetzung des Konzepts durch die Quoka AG schnell eine kritische Anzeigenmasse erreicht wird.

Der vorliegende Artikel ist wie folgt gegliedert: In Kapitel 2 erfolgt eine würdigende Betrachtung der Literatur zu der jungen Disziplin Market Engineering sowie zu elektronischen Immobilienbörsen. An diese Übersicht schließt sich die Darstellung der eingesetzten Methodik - Case Study als theoriestützendes Werkzeug - an. In Kapitel 4 werden aufbauend auf den Ergebnissen der Case Study die ersten vier Phasen des Market-Engineering-Prozesses exemplarisch durchlaufen. Eine Zusammenfassung sowie ein Ausblick auf weitere Forschungsschritte beschließen den Artikel.

\section{Einordnung in die Literatur}

Im folgenden Abschnitt wird eine kurze Auswahl der wichtigsten Literatur zur verwendeten Methodik des Market Engineering sowie aus den Bereichen der Internetökonomie und Immobilienmarktplätze, auf die die Methodik angewandt wird, präsentiert.

\subsection{Market Engineering}

Die noch sehr junge Disziplin Market Engineering fand ihre erste deutschsprachige Erwähnung in [Wein ${ }^{+}$03]. Neumann [Neum04] und Holtmann [Holt04] ist es mit ihren Werken gelungen, diese Disziplin in die bestehende Literatur einzubetten und einen umfassenden Bezugsrahmen für die weitere theoretische Ausarbeitung und praktische Nutzung des Market Engineering zu schaffen.

Mit seinem Grundlagenwerk zum Market Engineering leistet Neumann [Neum04] einen wichtigen Beitrag, um die Konturen der jungen Disziplin zu schärfen. Er unterscheidet in seiner Arbeit zwischen dem „Design-Objekt“ und dem „DesignProzess" und schafft damit einen Bezugsrahmen zur Klassifikation und Erstellung von elektronischen Märkten. Die Betrachtung eines Marktes als Design-Objekt wird ausgehend von der Beschreibung des Marktes als mikroökonomisches System um die beiden Sichtweisen Infrastruktur und Businessmodell erweitert. Im Rahmen des Design-Prozesses wird - ausgehend von der Mechanism Theory - der Market-Engineering-Prozess als strukturierte Vorgehensweise zur Planung und 
Erstellung von Märkten hergeleitet. Um das unstrukturierte Problem, elektronische Märkte zu gestalten, systematisch lösen zu können, unterteilt Neumann diesen Prozess in die vier Phasen „Environmental Analysis“, „Design and Implementation“, „Testing“ und „Electronic Market Introduction“. Durch diese Einteilung und die weitere Dekomposition der einzelnen Phasen wurde eine Methodik geschaffen, die es dem Market Engineering ermöglicht, die entstandenen Teilprobleme mit geeigneten Methoden zu lösen.

[Holt04] befasst sich mit der Organisation von Märkten und führt exemplarisch am Beispiel des elektronischen Wertpapierhandels den Market-EngineeringProzess durch. Im Fokus der Arbeit steht neben der integrierten Betrachtungsweise von ökonomischer, informationstechnischer und rechtlicher Sicht die Erstellung eines Bezugsrahmens zur strukturierten Erfassung von elektronischen Märkten. Dieses Framework begreift einen Markt als eine Menge institutioneller Regeln. Holtmann ordnet diese Regeln den drei Bereichen Marktmikro-, Infra- und Businessstruktur zu. Durch die Ausgestaltung der Regeln wird das Verhalten der Marktteilnehmer und somit auch das Marktergebnis beeinflusst. Basierend auf dem erstellten Bezugsrahmen betrachtet Holtmann den Markt nicht nur als Koordinationsmechanismus, sondern erweitert diese Sicht um die Perspektive, einen Markt als Produkt eines Dienstleistungsanbieters zu betrachten. Auf diesem Framework aufbauend wird der Market-Engineering-Prozess eingesetzt, um - unter Berücksichtigung der Interessen privater Investoren - systematisch einen innovativen Wertpapiermarkt zu gestalten.

\subsection{Internetökonomie und Immobilienmarktplätze}

Die Auswirkungen des Internets auf die Immobilienbranche werden in verschiedenen Fachbüchern umfassend diskutiert. Einen ganzheitlichen Überblick über verschiedene Facetten des e-Business und des Internet-Marketings in der Immobilienbranche vermitteln neben dem Beitrag von Picot et. al. [Pico $\left.{ }^{+} 01\right]$ das Werk von Rohmert und Böhm [RoBö01] sowie der Beitrag von Irsfeld [Irsf01].

Mit seinem Werk „Die grenzenlose Unternehmung“ leisten Picot et. al. einen wichtigen Beitrag zur Einordnung elektronischer Märkte in die Internetökonomie. Sie betonen besonders die starke Mediatisierung ${ }^{4}$ von Markttransaktionen. Eingebettet in einen ganzheitlichen Überblick über die Potenziale und Grenzen der Virtualisierung von Markt- und Unternehmensstrukturen stellen Picot et. al. die gesamte Marktaufgabe in Form von Koordinationsmodellen in den Vordergrund ihrer Betrachtung. Dabei messen sie auch der Art der Güter und Dienstleistungen, die auf einem elektronischen Markt gehandelt werden, eine hohe Bedeutung bei.

4 Hierunter wird die elektronische Abbildung der Kommunikationsbeziehungen zwischen den Marktteilnehmern [Pico ${ }^{+}$01, S. 338] verstanden. 
Somit kann nach Picot et. al. beispielsweise von einem elektronischen Markt für Bücher oder Immobilien gesprochen werden.

In Zusammenarbeit mit vielen Marktexperten und Vertretern des Immobilien-e-Business beleuchten Rohmert und Böhm [RoBö01] die nachhaltigen Auswirkungen des Internets auf das klassische Immobiliengeschäft. Als Resultat dieser langfristig ausgelegten Perspektive entwickeln sie Chancen für neue Geschäftsfelder und betrachten die Revolution der Geschäftsprozesse vom Bau über die Immobilienvermittlung und -beratung bis zur Entwicklung von Immobilienkapitalanlagen. Vor dem Hintergrund dieser Geschäftsprozesse werden die erfolgreichsten Unternehmen in den jeweiligen Marktsegmenten analysiert. Neben der Betrachtung der Potenziale, die solch ein Wandel mit sich bringt, analysieren Rohmert und Böhm auch die einsetzende Stagnation des Marktes und betrachten mögliche negative Auswirkungen des e-Business auf die Immobilienbranche.

Irsfeld [Irsf01] hingegen konzentriert sich auf das e-Marketing von Immobilien und umschreibt damit die nachhaltig marktorientierte Ausrichtung des Immobilienunternehmens und seiner e-Commerce-Aktivitäten im Internet. Weiterhin entwickelt er in seinem Werk aus den Anforderungen der Immobilieninteressenten und den veränderten Wettbewerbsbedingungen im Internet eine umfassende Internet-Strategie für professionelle Immobilienanbieter wie z. B. Makler.

\section{Methodik - Case Study zur Theoriestützung}

Fallstudien sind eine Forschungsmethodik aus der Soziologie, die jedoch auch in den Wirtschaftswissenschaften Anwendung findet. Sie wird vorwiegend als qualitative Methode eingesetzt [KiMe02, S. 37]. Da bei diesem Ansatz meist wenige Fälle individuell betrachtet und ausgewertet werden, wird diese Vorgehensweise auch als Einzelfallstudie bezeichnet. ${ }^{5}$,Das Ziel der Einzelfallstudie ist, genaueren Einblick in das Zusammenwirken einer Vielzahl von Faktoren ... zu erhalten, wobei sie meist auf das Auffinden und Herausarbeiten von typischen Vorgängen gerichtet ist.“ $\left[\mathrm{Fuch}^{+} 78\right.$, S. 181] Fallstudien dienen der ganzheitlichen Analyse von realen Phänomenen im Alltagskontext [Gass99, S. 11f] und werden eingesetzt, um ,in irgendeiner Weise typische, als extrem-, ideal oder durchschnittstypische Handlungsmuster zu identifizieren“ [Lamn89, S. 16].

Entsprechend der oben beschriebenen Zielsetzung kommen solche Studien zum Einsatz, wenn das ,wie“" und das ,warum“ untersucht werden sollen [Yin94, S. 6]. Im vorliegenden Artikel wird untersucht, wie (elektronische) Immobilienbörsen

5 In Abgrenzung dazu greift die quantitative Ausrichtung der Fallstudie auf ein großes erhobenes Datenvolumen zurück und zielt darauf $a b$, durch die Anwendung statistischer Methoden Generalisierungen abzuleiten. 
funktionieren, warum elektronische Immobilienbörsen dem herkömmlichen Anzeigenmarkt überlegen sind und wie eine zukunftsweisende elektronische Immobilienbörse zu gestalten ist.

Um diese Zielsetzung zu erreichen, wurde eine exploratorische Fallstudie durchgeführt, die als multiple case study mit konvergenter Auswertung [Yin94, S. 93] realisiert wurde. Zur Erhebung der notwendigen Daten wurden die Internetauftritte der sechs größten deutschen e-Immobilien-Marktplätze ${ }^{6}$ eingehend analysiert. Ergänzend wurden offene Interviews mit Maklern und mit Mitarbeitern der Quoka AG durchgeführt. Unter Einbezug der Erfahrung der Autoren bei der Wohnungssuche wurden die erhobenen Daten analysiert und dadurch ein umfassendes Bild über die bestehende Situation am Immobilienmarkt mit Fokus auf elektronische Immobilienmarktplätze und die Anforderungen der einzelnen auf diesem Markt auftretenden Teilnehmer erstellt. Diese Ergebnisse sowie die aus der Literaturarbeit gewonnenen Erkenntnisse bilden die theoretisch untermauerte, empirische Basis für die im folgenden Kapitel beschriebene Durchführung des MarketEngineering-Prozesses zur Erstellung eines internetbasierten Immobilienmarktplatzes.

\section{Market Engineering am Beispiel einer elektronischen Immobilienbörse}

Der folgende Abschnitt beschreibt die Durchführung des Market-Engineering-Prozesses zur Erstellung einer elektronischen Immobilienbörse. Als Domänenwissen wird auf die Ergebnisse aus der in Kapitel 3 beschriebenen Fallstudie zurückgegriffen. Nach einer kurzen Beschreibung des Market-Engineering-Prozesses in Abschnitt 4.1 wird in Abschnitt 4.2 anhand der ersten vier Phasen dieses Prozesses die Marktmikrostruktur des zu designenden Marktplatzes beschrieben.

\subsection{Beschreibung des Market Engineering Prozesses}

Wie Neumann in seiner Arbeit darstellt, muss bei der Gestaltung von Märkten zwischen dem Design-Objekt und dem Design-Prozess unterschieden werden [Neum04, S. 3]. Die Darstellung des Bezugsrahmens zur Erstellung des Design Objekts „elektronischer Markt“ in [Wein ${ }^{+} 03$, S. 637] verdeutlicht, dass bei der

6 Die Größe der Online-Marktplätze wurde anhand einer zusammengesetzten Messgröße ermittelt, die aus der Anzahl der Exposéabrufe pro Visit, der Anzahl der Objekte und den Page Impressions besteht. Folgende Marktplätze wurden in Rahmen der Case Study betrachtet: ImmobilienScout24, Immopool, Immowelt, 1AImmobilienmarkt, PlanetHome, Immonet. 
Gestaltung und Betrachtung von elektronischen Märkten von dem tatsächlichen, also dem sozioökonomischen, technischen und rechtlichen Umfeld ausgegangen werden muss (vgl. Abbildung 1). Dieses Umfeld liegt ebenso wie das Verhalten der Marktteilnehmer nicht im Einflussbereich des Market Engineer. Neben der adäquaten Abbildung des Transaktionsobjekts besteht die Aufgabe des Market Engineer darin, die Transaktionsdienstleistung zu gestalten. Deren Ausgestaltung hat signifikanten Einfluss auf das Verhalten der Marktteilnehmer und damit auch auf das Marktergebnis und die Marktqualität [Smit82, S. 923]. Abbildung 1 verdeutlicht diesen Zusammenhang und stellt die von Holtmann in seiner Arbeit beschriebene Dreiteilung der Transaktionsdienstleistung in die Marktmikrostruktur, die Infrastruktur und die Businessstruktur dar [Holt04, S. 152ff].

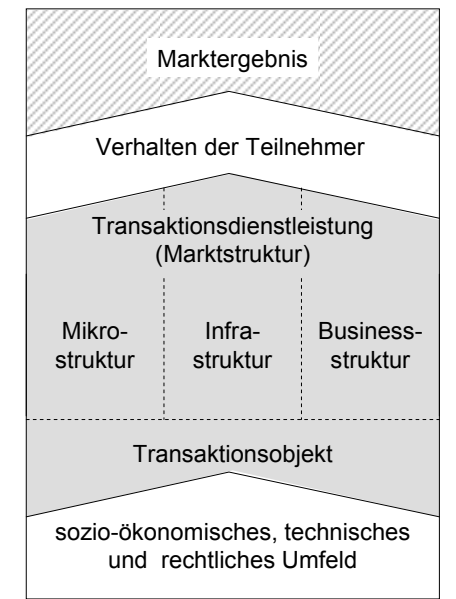

Abbildung 1: Komponenten eines (elektronischen) Marktes $^{7}$

Die Gestaltung dieser drei Säulen der Marktstruktur erfolgt anhand des iterativen Market-Engineering-Prozesses (vgl. Abbildung 2). ${ }^{8}$ Die ersten beiden Gestaltungsschritte dieses Prozesses setzen sich dabei aus der Stakeholderidentifikation und der Anforderungsanalyse zusammen. Während der sich anschließenden Transaktionsobjektgestaltung wird in Phase 3 das $\mathrm{zu}$ handelnde Gut strukturiert erfasst. Basierend auf den Ergebnissen der ersten drei Phasen folgt in Phase 4 die Transaktionsdienstleistungsgestaltung. Den Abschluss des Market-EngineeringProzesses bilden die Phasen 5 und 6. Erstere beinhaltet mit der Implementierung, dem Testen und einer abschließenden Evaluation die Umsetzung des erstellten Konzepts. Der Betrieb des Marktes sowie die für den erstmaligen Betrieb notwendigen Schritte erfolgen in Phase 6.

7 In Anlehnung an Abbildung 2 in [Wein ${ }^{+}$03, S. 637].

$8 \quad$ Neumann bezeichnet dies als Design-Prozess [Neum04, S. 155]. 


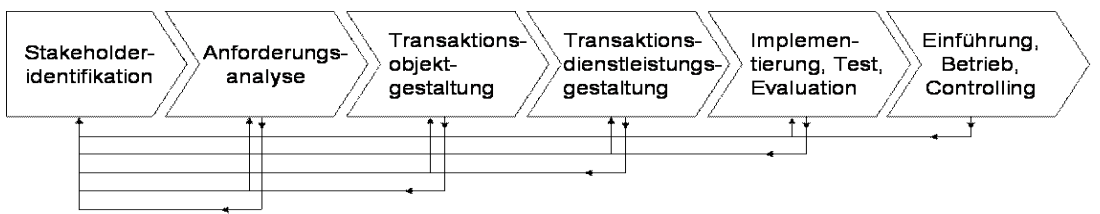

Abbildung 2: Market-Engineering-Prozess ${ }^{9}$

\subsection{Umsetzung des Market-Engineering-Prozesses}

\subsubsection{Stakeholder Identifikation (Phase 1)}

Die Teilnehmer des Wohnimmobilienmarktes lassen sich neben dem Plattformbetreiber $^{10}$ in Nachfrager- und Anbietergruppen unterteilen [Perl02, S. 23]. Die Gruppe der Anbieter wird weiterhin nach gewerblichen (z. B. Bauträger, Makler) und nach privaten Inserenten unterschieden. Diesen gegenüber stehen die Nachfrager, die das Immobilienangebot nach ihren individuellen Wunschkriterien durchsuchen. Die Gruppe der Nachfrager besteht vorwiegend aus privaten Haushalten. Die letzte Stakeholder-Gruppe, die auf einer elektronischen Immobilienbörse tätig ist, umfasst die Servicepartner, die bspw. Content für Zusatzservices der Immobilienbörse liefern oder als Werbepartner fungieren.

Im Hinblick auf den Umsatz der Immobilienbörse kann eine Gewichtung der einzelnen Gruppen erfolgen. Dabei zeigt sich, dass auf den bereits bestehenden internetbasierten Immobilienmärkten ca. 90\% aller Anzeigen von gewerblichen und nur ca. $10 \%$ von privaten Anbietern stammen. Aufgrund dieser Tatsache ist für den Plattformbetreiber die Gruppe der gewerblichen Anbieter besonders interessant, da sie letztlich den meisten Umsatz generiert.

\subsubsection{Anforderungsanalyse (Phase 2)}

Aufbauend auf der Erfassung der Stakeholder werden in Phase 2 die Anforderungen der Marktteilnehmer an eine elektronische Immobilienbörse erfasst.

9 In Anlehnung an Abbildung 3 in [Wein ${ }^{+}$03, S. 639].

10 Kunzelmann et. al. unterscheiden zwischen Plattform- und Marktplatzbetreiber. Der Plattformbetreiber ist für den reibungslosen technischen Betrieb der Plattform sowie für die Einhaltung juristischer Rahmenbedingungen zuständig. $\mathrm{Er}$ trägt das ökonomische Risiko der Plattform. Dem gegenüber ist der Marktplatzbetreiber für die Ausgestaltung der Marktstruktur des von ihm auf der Plattform betriebenen Marktplatzes zuständig [Kunz ${ }^{+}$04, S. 86f]. In der vorliegenden Arbeit fallen die Rollen Plattform- und Marktplatzbetreiber für den Bereich der privaten Inserate zusammen. Die teilnehmenden Makler werden als eigenständige Marktplatzbetreiber angesehen, da sie einen eigenen Bereich auf der Plattform haben. 


\section{Plattformbetreiber}

Für den Plattformbetreiber ist es als Eigentümer der Immobilienbörse von größtem Interesse, den Erfolg derselben sicher zu stellen. Hierfür benötigt er neben einem attraktiven Serviceangebot eine große Anzahl von Anbietern und Nachfragern. Um für die gewerblichen Anbieter die Teilnahme an der Immobilienbörse attraktiv zu gestalten, muss diesen ermöglicht werden, innerhalb der Plattform ihren eigenen Bereich individuell zu gestalten. Hieraus folgt, dass die Immobilienbörse aus softwaretechnischer Sicht mandantenfähig sein muss [Kunz 04, S. 87].

\section{Gewerbliche Anbieter}

Das Hauptziel, das ein gewerblicher Immobilienanbieter mit der Präsentation seiner Objekte im Internet verfolgt, ist die Ansprache potentieller Kunden. Als Instrument der Kaufanbahnung dienen elektronische Immobilienbörsen dazu, diese Kunden zu erreichen und zu informieren [RoBö01, S. 222]. Neben der geforderten Mindestgröße des Marktplatzes sind die drei wichtigsten Kernkompetenzen, die nach Angabe der gewerblichen Anbieter erfüllt sein müssen, die technische Zuverlässigkeit der Immobilienbörse, die zielgenaue Ansprache von Kundensegmenten und ein attraktives Preis-Leistungsverhältnis [RoBö01, S. 83].

- Unter der technischen Zuverlässigkeit wird dabei das komfortable und sichere Übertragen der Immobilienangebote in die jeweilige Börse verstanden. Hierzu sind weiterhin Schnittstellen zu gängiger Maklersoftware nötig.

- Die zielgenaue Ansprache der verschiedenen Kundensegmente ist ebenfalls eine Kernfunktion, die jedes Immobilienportal anbieten sollte. Diese Funktion stellt in gewisser Weise sogar die wichtigste aller Anforderungen dar, da es entscheidend für den Anbieter ist, inwieweit die Immobilienbörse in der Lage ist, potenzielle Kunden zu erreichen, die evtl. später einen Kauf- oder Mietvertrag unterschreiben. Eingebettet in ein ganzheitliches Customer Relationship Management wird der Kunde mit Hilfe der Value Added Services bei seiner Kauf- bzw. Mietentscheidung begleitet [RoBö01, S. 81f].

Neben der Gliederung des Objektangebots in verschiedene Unterrubriken findet sich ein weiteres Mittel, um Kunden gezielt zu werben, in einer strukturierten und detaillierten Objektbeschreibung. Diese Beschreibung unterstützt den Immobilieninteressenten bei seiner Suche und liefert ihm - je nach seinen individuellen Wunschkriterien - die passende Immobilie. Sie dient weiterhin zur Betonung spezieller Ausstattungsmerkmale oder Besonderheiten der Immobilie. Zusätzlich kann diese Funktion durch eine geeignete Objektplatzierung innerhalb der Website (z. B. auf der Startseite als Highlight) oder durch den Einsatz von Value Added Services unterstützt werden.

- Als letzte der aufgeführten Kernkompetenzen ist das Preis-Leistungsverhältnis zu nennen. Hierbei fordert der gewerbliche Anbieter ein flexibles und möglichst günstiges Preismodell, das z. B. gestaffelt nach der Anzahl der Anzeigen gewisse Preisnachlässe oder zusätzliche Services vorsieht. Weiterhin sollte ei- 
ne nachvollziehbare und unkomplizierte Abrechnung erfolgen, die eventuell erfolgsbasiert (z. B. anhand der erzielten Kundenkontakte) umgesetzt werden kann.

\section{Private Anbieter}

Die Anforderungen der gewerblichen Anbieter an einen elektronischen Immobilienmarkt können ohne Probleme auch auf die privaten Anbieter übertragen werden. Im Unterschied zu dem Bedarf der gewerblichen stellen bei den privaten Anbietern die technische Zuverlässigkeit und das Preis-Leistungsverhältnis die größten Anforderungen an den Marktplatz dar. Ein weiterer Anspruch der privaten Anbieter adressiert die unproblematische Einstellung und Verwaltung der Objekte sowie die bereits erwähnte Mindestgröße der Immobilienbörse. Damit das private Inserat nicht in der Masse der kommerziellen Anzeigen untergeht, wäre außerdem die Möglichkeit einer expliziten Abgrenzung zu den gewerblichen Anbietern wünschenswert.

\section{Nachfrager}

Die beiden wichtigsten Anforderungen der Nachfrager sind bequeme Suchmöglichkeiten und ein breites Objektangebot [ObWi02]. Letzteres ist, insb. aufgrund der damit verbundenen Netzeffekte, mit die wichtigste Voraussetzung für den Erfolg einer Immobilienbörse. Die Qualität der Suchoptionen und eine hohe regionale Trefferzahl [ObE100, S. 106f] sind für den Nachfrager ebenfalls wichtige Kriterien, da ein großes Objektangebot nicht zwangsläufig die gewünschte Trefferzahl und -qualität garantiert. Neben den gängigen Suchmöglichkeiten (Standort, Preis, Wohnfläche) und aufgrund des regionalen Angebotsüberhangs [Perl02, S. 8] werden qualitative Objektmerkmale (z. B. Luxusausstattung wie ein Kamin oder eine Sauna) für den Nachfrager immer wichtiger [Brau03, S. 20]. Um all diese Wünsche und Kriterien zu berücksichtigen, wird folglich eine innovative Suchfunktion benötigt. Sie muss sowohl Interessenten, die noch keine genauen Vorstellungen von ihrer Wunschimmobilie haben, als auch Nachfrager, die z. B. bestimmte Ausstattungsmerkmale oder eine bestimmte Lage innerhalb einer (evtl. eng umrissenen) Region wünschen, bedienen. Als Ergebnis erwartet der Interessent eine übersichtliche und umfassende Objektpräsentation, die sowohl einen visuellen Eindruck vermittelt, als auch eine detaillierte Objekt- und Lagebeschreibung enthält. Neben einer umfassenden Suchfunktion wünschen die Nachfrager weitergehende Sortier- und Selektiermöglichkeiten.

Ein weiterer Service, der von Nachfragern gefordert wird, ist die Speicherung bereits getätigter Suchanfragen. Wird nach Speichern der Suchanfrage ein neues Objekt, das diesen Anforderungen gerecht wird, in die Datenbank eingestellt, kann der Interessent proaktiv auf diese Immobilie hingewiesen werden.

Neben den reinen Objektinformationen erwartet der Interessent eine Komplettbetreuung auf dem Weg vom Exposé zum tatsächlichen Kauf der Immobilie. Diesen Weg unterstützen bestimmte Zusatzservices und Dienstleistungsangebote, die 
sich z. B. auf die Finanzierung der Immobilie oder auf den anstehenden Umzug spezialisiert haben.

\subsubsection{Transaktionsobjektgestaltung (Phase 3)}

Ausgangspunkt für den Erfolg einer elektronischen Immobilienbörse ist die geeignete Erfassung der Transaktionsobjekte in einer Datenbank. Hierfür gilt es, die Immobilien trotz deren großer Heterogenität in einer einheitlichen Struktur und standardisiert abzubilden. ${ }^{11}$ Dies ermöglicht datenbankgestützte Eingabe- und Abrufmöglichkeiten und gewährleistet damit eine komfortable Nutzung der Immobilienbörse - sowohl für Nachfrager als auch für Anbieter.

$\mathrm{Da}$ in der Literatur keine Ansätze zu einer strukturierten, ganzheitlichen und anwendungsorientierten, systemtechnischen Abbildung von Immobilien vorhanden sind, wird die Transaktionsobjektgestaltung aufbauend auf den Ergebnissen der Case Study entwickelt.

Bei der Erfassung der Attribute von Wohnimmobilien bietet es sich aufgrund der z. T. unterschiedlichen Eigenschaften an, zwischen den Grundtypen Haus und Wohnung zu unterscheiden. So ist es bei einer Wohnung beispielsweise von Interesse, wie viele weitere Wohneinheiten sich im Haus befinden, bzw. wo im Haus sich die Wohnung befindet, während bei Häusern die Größe des Grundstücks oder Details wie Wegerechte von Bedeutung sind. Da sich die Attributstruktur der Objekte in Abhängigkeit davon, ob es sich um Miet- oder Kaufobjekte handelt, kaum unterscheidet, werden alle Objekte des gleichen Grundtyps in einer Tabelle erfasst.

Tabelle 1 verdeutlicht exemplarisch einige wohnungsbeschreibende Attribute. Neben der Nennung des Attributs und einer Auflistung möglicher Ausprägungen beinhaltet die Tabelle Informationen darüber, ob das Ausfüllen des Feldes bei der Objekterfassung verpflichtend ist, ob das Attribut mehrere Ausprägungen annehmen kann und ob die Information zu mietende bzw. zu kaufende Wohnungen betrifft.

Neben der skizzierten Erfassung der Objekteigenschaften und finanzieller Aspekte bedarf es für die vollständige Beschreibung einer Immobilie, die eine Bereitstellung von Value Added Services bzw. die automatische Erstellung eines vollständigen Exposés ermöglicht, der Aufnahme weiterer Attribute bzw. des Einbezugs weiterer Daten. Die einzelnen eine Immobilie beschreibenden Attribute lassen sich jeweils einer der folgenden Kategorien zuordnen: beschreibende Objektdaten, $f i$ nanzielle Objektdaten, Adresse des Objekts, Lage des Objekts, Freizeitwert, Verkehrsanbindung.

11 Bei Immobilien ist dies in Analogie zu Pkws bzw. Stellenanzeigen möglich. Dem gegenüber sind andere Kleinanzeigenrubriken wie Haushaltsgeräte oder Möbel weniger geeignet, um sie jeweils in einer einheitlichen Struktur zu erfassen. 
Während die Objektdaten sowie die Adresse des Objekts vom Anbieter einzupflegen sind, können Informationen zur Lage des Objekts ${ }^{12}$, zu dessen Freizeitwert ${ }^{13}$ und Verkehrsanbindung anhand der exakten Adresse des Objekts automatisch generiert werden.

\begin{tabular}{|l|l|l|l|l|}
\hline Attribut & Ausprägungen & $\begin{array}{l}\text { Ver- } \\
\text { pflich- } \\
\text { tend }\end{array}$ & $\begin{array}{l}\text { Mehrfach- } \\
\text { belegung } \\
\text { möglich }\end{array}$ & Miete / Kauf \\
\hline $\begin{array}{l}\text { Anzahl der } \\
\text { Zimmer }\end{array}$ & $\begin{array}{l}\text { 1,2, 3, 4, 5 oder mehr } \\
\text { Zimmer }\end{array}$ & ja & nein & Miete / Kauf \\
\hline $\begin{array}{l}\text { Lage innerhalb } \\
\text { des Wohn- } \\
\text { hauses }\end{array}$ & $\begin{array}{l}\text { Dachgeschoß, Erdge- } \\
\text { schoß, Keller, 1., 2., 3., } \\
\text { n-ter Stock }\end{array}$ & ja & nein & Miete / Kauf \\
\hline $\begin{array}{l}\text { Wohnungstyp } \\
\text { Maisonette, Penthouse, } \\
\text { Loft, Studio, ... }\end{array}$ & nein & nein & Miete / Kauf \\
\hline $\begin{array}{l}\text { Ausstattungs- } \\
\text { typ }\end{array}$ & $\begin{array}{l}\text { Behindertengerecht, } \\
\text { Rollstuhlgerecht, Seni- } \\
\text { orengerecht, Nichtrau- } \\
\text { cher, ... }\end{array}$ & nein & ja & Miete / Kauf \\
\hline $\begin{array}{l}\text { Luxusinnen- } \\
\text { ausstattung }\end{array}$ & $\begin{array}{l}\text { Wintergarten, Lift, } \\
\text { Sauna, Kamin, Pool, ... }\end{array}$ & nein & ja & Miete / Kauf \\
\hline Preis & "Betrag in $\epsilon^{\prime \prime}$ & ja & nein & Miete / Kauf \\
\hline Wohnfläche & "Größe in $m^{2}$ “ & ja & nein & Miete / Kauf \\
\hline ‥ & $\ldots$ & $\ldots$ & $\ldots$ & ... \\
\hline Renovierung & beim Einzug / Auszug & nein & nein & Miete \\
\hline Courtage & $\begin{array}{l}\text { Anzahl der Kaltmieten / } \\
\text { mo vom Wert der Im- } \\
\text { mobilie }\end{array}$ & ja & nein & Miete / Kauf \\
\hline Notargebühr & "Betrag in $\epsilon^{\prime \prime}$ & nein & nein & Kauf \\
\hline
\end{tabular}

Tabelle 1: Attribute einer Wohnung

\subsubsection{Transaktionsdienstleistungsgestaltung (Phase 4)}

Die Gestaltung der Transaktionsdienstleistung steht im Mittelpunkt des MarketEngineering-Prozesses. Sie baut auf den vorangegangenen Phasen auf und setzt

Die Lage des Objekts umfasst beispielsweise Informationen über die Nähe zu Ärzten, Apotheken oder Einkaufmöglichkeiten.

13 Der Freizeitwert einer Immobilie kann durch die Existenz von Naherholungsgebieten, wie auch durch die Nähe zu Restaurants oder einem Theater beschrieben werden. 
die Spezifikation des Transaktionsobjektes in Verbindung mit der Anforderungsanalyse in der Beschreibung der Marktstruktur um. Diese Struktur umfasst drei Säulen, die Marktmikrostruktur, die Infrastruktur und das Businessmodell, wobei im Folgenden ausschließlich auf die Marktmikrostruktur eingegangen wird.

Die Beschreibung der Marktmikrostruktur kann entweder anhand von Regeln, auf Basis einer Taxonomie oder durch eine rein sprachliche Darstellung erfolgen. Bei der Auswahl des geeigneten Beschreibungstyps kommt den formalen Anforderungen und dem anvisierten Design des elektronischen Marktes besondere Bedeutung zu. Der vorliegende Markt unterstützt aufgrund der Objekteigenschaften der Immobilie [Schu98, S. 18ff] und der juristischen Rahmenbedingungen ${ }^{14}$ ausschließlich die Informationsphase. ${ }^{15}$ Märkte, die zusätzlich die Vereinbarungs- und Abwicklungsphase umfassen, wären z. B. der elektronische Wertpapierhandel und Online-Auktionen elektronischer Güter. Im Gegensatz $\mathrm{zu}$ dem sehr heterogenen Gut Immobilie erfolgt die Zusammenführung von Angebot und Nachfrage bei Aktien lediglich über deren Preis. Ein elektronischer Immobilienmarkt verfügt nicht über einen vergleichbaren Preisfindungsmechanismus. Stattdessen findet eine unverbindliche Suche statt, in deren Zentrum das Transaktionsobjekt - die Immobilie - steht. Aufgrund der klaren Zuordnung zur Informationsphase wurde bei der Modellierung der Marktmikrostruktur auf die sprachlich beschreibende Variante zurückgegriffen.

Die Zugangssituation der Handelsteilnehmer zum Markt gestaltet sich offen $\left[\mathrm{Pico}^{+} 01\right.$, S. 340ff]. Jeder Immobilieninteressent und -anbieter kann den elektronischen Immobilienmarkt im Internet besuchen und nach einer geeigneten Immobilie suchen bzw. diese anbieten. Dabei treffen - wie bereits erwähnt - Immobilieninteressenten oder -nachfrager auf Immobilienanbieter. Auf beiden Seiten können beliebig viele Teilnehmer auftreten und Inserate einstellen [StWe03, S. 152]. ${ }^{16}$ Eine zeitliche Beschränkung durch die Marktmikrostruktur hinsichtlich der zeitlichen Nutzbarkeit des Marktes erfolgt ausschließlich durch die Vorgabe der Gültigkeitsdauer der Anzeigen. Der Zugriff auf die Immobilienangebote aus Sicht des Nachfragers ist jederzeit möglich. Er unterliegt keinerlei zeitlichen Beschränkungen.

Für die Verfügbarkeit des Dienstleistungsangebots ist, ebenso wie für die Betreuung der Kunden und evtl. notwendige Regulierungsschritte, der Plattformbetreiber ${ }^{17}$ zuständig [Kunz ${ }^{+}$04, S. 89f]. Die zentrale Dienstleistung, die auf dem elektronischen Immobilienmarkt angeboten wird, ist die Erstellung von und Suche

14 Der Kauf bzw. Verkauf von Immobilen bedarf in Deutschland bspw. der Einbindung eines Maklers [Murf97, S. 739ff].

15 Zur systematischen Unterteilung des Handels auf elektronischen Märkte in die Informations-, die Vereinbarungs- und die Abwicklungsphase vgl. [Schm93, S. 467].

Es liegt also eine "m:n"- Beziehung vor.

17 Kunden sind aus Sicht des Plattformbetreibers in erster Linie Makler und Immobilieninserenten, aber auch Contentprovider und Werbepartner zählen zu den Kunden. 
nach Immobilienangeboten. Die Suche erfolgt über die Gesamtzahl der angebotenen Immobilien und zeigt als Ergebnis diejenigen Objekte an, die den jeweiligen Such- bzw. Wunschkriterien des Immobilieninteressenten entsprechen. Die Umsetzung dieser Funktionalität baut auf der Transaktionsobjektgestaltung auf, die zur Erfassung der einzelnen Beschreibungsmerkmale und deren Attributausprägungen dient. Zur Eingabe des Objekts bzw. der Suchanfrage wird der Kunde iterativ durch den Eingabe- bzw. Suchprozess geleitet. ${ }^{18}$ Dabei erfolgt zuerst eine allgemeine Abfrage, die schließlich - je nach Interesse des Kunden - immer detaillierter wird. Beispiele für allgemeine Objektdaten sind der Wohnimmobilientyp, die Vertragsart (Kauf oder Miete) sowie der gewünschte Standort, die Größe und die Preisklasse des Objekts. Zusätzlich kann eine Detailsuche erfolgen, die weitere bauliche Aspekte oder ergänzend finanzielle und rechtliche Suchkriterien enthält. Weitere Abfragepunkte beziehen sich auf die allgemeine Objektlage sowie auf den Verkehrswert, den Freizeitwert und die Lage zu medizinischen oder sozialen Einrichtungen in der Objektumgebung. Letztlich hat der Immobilieninteressent die Möglichkeit, individuell gewünschte Ausstattungsmerkmale zu selektieren, die z. B. separat als Luxusausstattungsmerkmale zur Auswahl stehen.

Als Ergebnis der Suchanfrage erhält der Immobilieninteressent ein oder mehrere Exposés bzw. Objektpräsentationen, die sowohl eine grafische als auch eine schriftliche Objektbeschreibung enthalten. Die spezifizierte Abfrage wird weiterhin in der Datenbank hinterlegt, sodass der Wohnungssuchende auf Basis der hinterlegten Suchfunktion auf passende neu eingestellte Objekte hingewiesen werden kann.

Die Einstellung eines Objekts in die Immobilienbörse erfolgt analog zur Spezifikation der Suchfunktion. Wie in Abbildung 3 dargestellt, wird den Anbietern die Möglichkeit gegeben, ihre Objektbeschreibung strukturiert anhand der genannten Kriterien einzustellen.

Rund um diese Kerndienstleistung existieren noch weitere Services und Angebote, die einer aktiven Gestaltung bedürfen. Basierend auf der Anforderungsanalyse der einzelnen Stakeholder treten drei weitere, nachfolgend beschriebene Serviceangebote bzw. ergänzende Dienstleistungen in den Vordergrund, die einer bewussten und zielgerichteten Gestaltung bedürfen.

18 Da die Erfassung von Objekten und Suchanfragen sehr ähnlich ist, wird im Folgenden ausschließlich die Eingabe einer Suchanfrage beschrieben. 


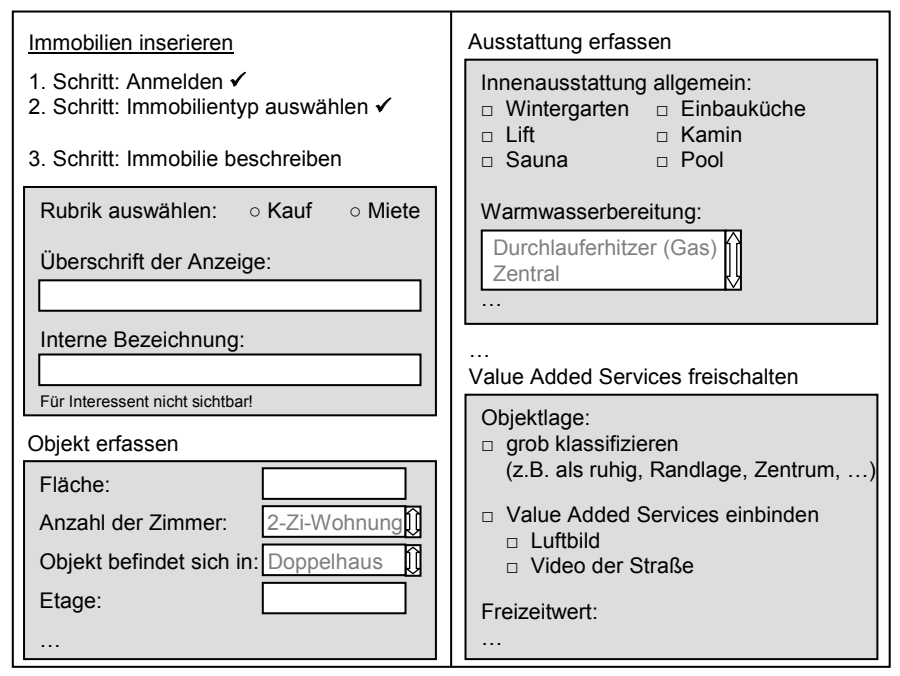

Abbildung 3: Beispiel für die Eingabe einiger Attribute einer Wohnung

\section{Übersichtliche und umfassende Objektpräsentation}

Die Präsentation einer Immobilie, die der Immobiliennachfrager bzw. -interessent als Ergebnis seiner Suchanfrage erhält, wird in Form eines elektronischen Exposés dargestellt. Um dem Kunden sofort einen visuellen Bezug zur Immobilie zu geben, erfolgt zunächst die Darstellung anhand verschiedener Ansichten, Grundrisse, Luftbilder, Stadtpläne und sonstiger Visualisierungstechniken (Virtuelle Begehung, $360^{\circ}$-Aufnahmen, 3D-Animation). Zusätzlich werden die wichtigsten Objektdaten kompakt und tabellarisch aufbereitet präsentiert (vgl. Abbildung 4). Für weitergehende Informationen stehen ein Detail-Exposé sowie weitere Value Added Services wie Umgebungskarten, Umfeld- und Shoppinganalysen, Ortsinformationen oder Soziostrukturdaten zur Verfügung. Auch ein sofortiger unverbindlicher Kontakt zum Anbieter und dessen weiteren Angeboten ist innerhalb des Exposés gegeben.

\section{Value Added Services}

Value Added Services stellen eine Möglichkeit dar, sich von anderen Immobilienmärkten und -anzeigen abzuheben und dem Kunden einen Mehrwert zu bieten. Ihnen kommt entlang der kompletten Wertschöpfung eines elektronischen Immobilienmarktes eine wesentliche Bedeutung zu. Neben der im vorherigen Punkt beschriebenen Möglichkeit, Immobilien repräsentativ darzustellen, können der Objektbeschreibung ergänzende Standortinformationen beigefügt werden. Diese liefern anhand des exakten Objektstandorts wirtschaftliche, soziale oder freizeit- und infrastrukturrelevante Zusatzinformationen. Die Umsetzung selbst erfolgt durch den Einkauf dieser Daten von öffentlichen [FoOe02, S. 16ff] und privaten Anbietern. Neben diesen direkt auf ein Objekt bezogenen Value Added Services können 
Wohnungssuchenden in Anlehnung an Automobilbörsen weitere Dienstleistungen wie Finanzierungsofferten, Checklisten für die Wohnungsauswahl oder Kontaktdaten zu evtl. benötigten Dienstleistungsanbietern, wie Notaren oder Sachverständigen angeboten werden [Euro02, S. 15]. Ein weiterer zusätzlicher Nutzen ergibt sich durch die Einrichtung persönlicher Bereiche, die sowohl inhaltlich als auch strukturell umgesetzt werden. Ziel dieser Personalisierbarkeit ist es, den Kunden persönlich anzusprechen und ihm einen eigenen Bereich mit seinen individuell benötigten Funktionen innerhalb der Website anzubieten. Solche Funktionen sind beispielsweise die Verwaltung der Anzeigen und der entstandenen Kosten oder die Speicherung der persönlichen Daten und der bereits getätigten Suchanfragen.

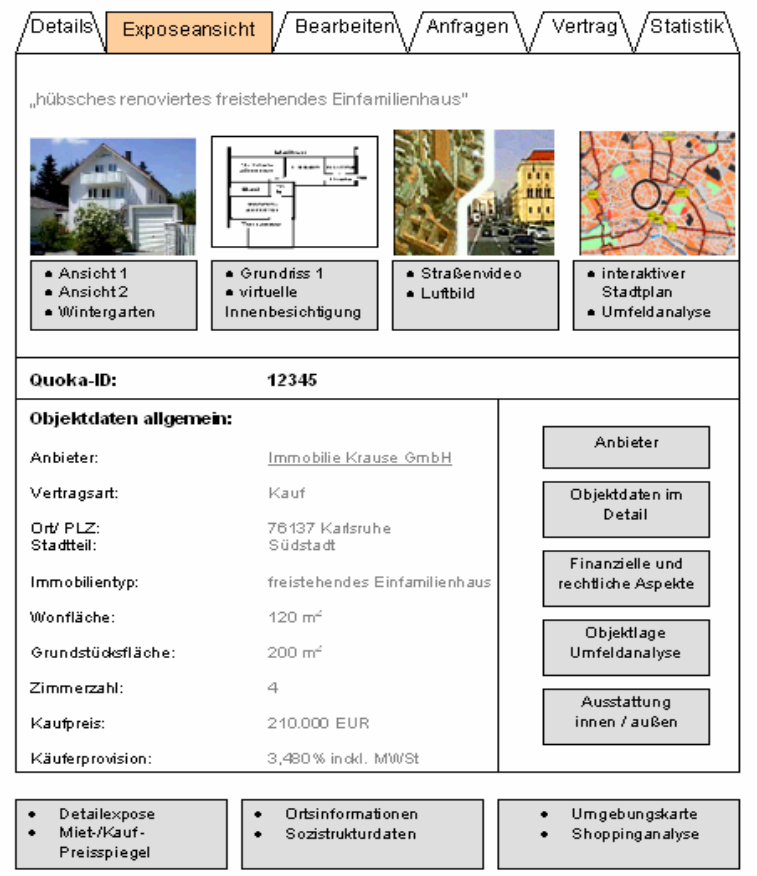

Abbildung 4: Beispielexposé auf der konzipierten Immobilienbörse

\section{Berücksichtigung gewerblicher Anbieter}

Gewerbliche Anbieter, die sich vorwiegend aus Maklerunternehmen zusammensetzen, erbringen z. Z. auf den Online-Immobilienbörsen den größten Teil des Umsatzes. Neben den bereits beschriebenen Services sollte Maklern eine individuelle Unternehmenspräsentation innerhalb des elektronischen Marktes angeboten werden. ${ }^{19}$ Hier kann das Maklerunternehmen ein eigenes Corporate Design umset-

19 Vgl. zu dieser Funktionalität auch die Seiten der Automobilhändler bei mobile.de. 
zen und alle seine Angebote kombiniert mit einer Unternehmensvorstellung präsentieren. Neben der Auflistung seiner Verbandsmitgliedschaft und seiner Tätigkeitsschwerpunkte erhält der Makler von Jobbörsen übernommene Verwaltungsfunktionen wie Abrufstatistiken für die jeweiligen Objekte oder ein Postfach für eingegangene Anfragen bzw. Kontakte. Als Erfolgsnachweis für den Kunden könnte er zusätzlich positive Kundenbewertungen mit einbinden und bereits verkaufte bzw. vermietete Objekte hervorheben, die qualitativ sehr hochwertig sind.

Grundsätzlich werden mit der detaillierten Erfassung von Immobilien, der Möglichkeit, sehr genaue Suchanfragen zu spezifizieren, sowie mit der Erfüllung der drei beschriebenen Punkte die wichtigsten Erfolgsfaktoren eines elektronischen Immobilienmarktes erfüllt. Von besonderer Bedeutung für den Erfolg ist die Integration von privaten und gewerblichen Anzeigen auf einem Marktplatz, da auf diesem Weg die für eine Konsolidierung notwendige kritische Masse erreicht werden kann. Um diese aus der gesteigerten Reichweite resultierenden Netzeffekte $\mathrm{zu}$ erzielen, bedarf es einer fehlerfreien Leistungserbringung sowie einer hohen technischen Zuverlässigkeit der Plattform. Neben dieser Anforderung an die Infrastruktur sollte ein flexibles und leistungsgerechtes Abrechnungsmodell [Pico ${ }^{+} 01$, S. 366f] bzw. ein attraktives Preis-Leistungsverhältnis angeboten werden. Durch eine solche gesamtheitliche Gestaltung der Mikro-, Infra- und Businessstruktur werden optimale Bedingungen für den Erfolg der konzipierten Immobilienbörse geschaffen.

\section{Fazit und Ausblick}

Ausgehend von dem aktuellen Trend weg von Print-Kleinanzeigen und hin zu internetbasierten domänenspezifischen Marktplätzen wurde eine Case Study über bestehende elektronische Immobilienbörsen durchgeführt. Basierend auf den Ergebnissen der Studie wurde unter Nutzung des Market-Engineering-Prozesses die Marktmikrostruktur einer zukunftsweisenden Online-Immobilienbörse erstellt. Die Umsetzung dieser Struktur durch die Quoka AG ist geplant. Dabei wird das Ziel verfolgt, durch die gesteigerte Attraktivität des Immobilienmarktplatzes die notwendige kritische Masse an Anbietern und Nachfragen zu erreichen, um so eine Konsolidierung in dieser Domäne einzuleiten. Trotz der anfänglichen Fokussierung auf den süddeutschen Raum sind die erzielten Ergebnisse auf andere Regionen bzw. Länder übertragbar, da die Objektstruktur und die Anforderungen der Stakeholder hiervon unabhängig sind.

Neben dem Ziel einer Konsolidierung der privaten Immobilienangebote und -gesuche wird als deutliche Abgrenzung und funktionale Erweiterung zu bestehenden Online-Immobilienmärkten die Integration des Direkt- und des Maklermarktes verfolgt. Dabei wird es Maklern ermöglicht, ihre Immobilien auf der Immobilienbörse innerhalb ihres eigenen Shops individuell zu präsentieren. Woh- 
nungssuchende können sich bei der Spezifikation ihrer Anfrage entscheiden, ob ihnen nur Objekte privater oder nur Objekte kommerzieller Anbieter oder beide Gruppen von Objekten angezeigt werden. ${ }^{20}$

Aufgrund des hohen Anteils an Fixkosten, die beim Betrieb einer internetbasierten Immobilienbörse anfallen, können mit wachsender Teilnehmerzahl zunehmend Economies of Scale realisiert werden. Die erwartete wachsende Teilnehmerzahl führt durch die mit dem Betrieb eines Online-Marktplatzes verbundenen Netzeffekte [Econ96, S. 677f] weiterhin zu einer zunehmenden Attraktivität der Immobilienbörse. Durch einen möglichen Erfolg des konzipierten Immobilienmarktplatzes und die damit einhergehende Konsolidierung ist eine erhebliche Steigerung der Markttransparenz [ObEl00, S. 38] zu erwarten. Diese kann neben einer Stärkung des Wettbewerbs zu einer erheblichen Reduktion der Such- bzw. Informationskosten der Nachfrager führen [Jenk96, S. 25]. Weiterhin kann durch die Erfassung und Auswertung der Suchanfragen Einblick in die Nachfrage gewonnen werden, sodass Makler gezielt gefragte Immobilien in ihr Angebot aufnehmen können.

Vor der Implementierung der konzipierten Marktmikrostruktur müssen die Infraund die Businessstruktur erstellt werden. Während die Anforderungen an die Infrastruktur parallel zur Konzeption der Mikrostruktur erfasst wurden, bedarf die Erstellung des Businessmodells einer eingehenden Betrachtung. Dabei gilt es insb. die Zahlungsbereitschaft der Makler sowie der privater Marktteilnehmer zu evaluieren. Weiterhin sind für die unterschiedlich aufwendige Präsentationen der Objekte und deren Ausstattung mit Zusatzservices verschiedene Gebührenmodelle zu entwickeln.

Im Hinblick auf den späteren Betrieb der Immobilienbörse ist zur Messung der ökonomischen Rentabilität die Konzeption und Umsetzung eines geeigneten IuKControllings unerlässlich. Neben der Auswertung der Geschäftsbeziehungen mit den privaten Marktteilnehmern sind insb. die Beziehungen zwischen dem Plattformbetreiber und den Maklern, die jeweils als eigenständige Marktplatzbetreiber agieren, von Interesse. Eine entsprechende Erfassung des Customer Lifetime Values der einzelnen Makler aus Sicht des Plattformbetreibers kann zur Sicherung und Intensivierung der Geschäftsbeziehung eingesetzt werden.

20 Die Möglichkeit Gebote nur in bestimmten Teilen eines Marktes anzuzeigen, wird von Neumman et. al. in $\left[\mathrm{Neum}^{+} 02\right.$, S. 298] als kaskadierend dynamisches Marktmodell bezeichnet. 


\section{Literatur}

[Brau03] Brauer, K.-U.: Grundlagen der Immobilienwirtschaft: Recht, Steuern, Marketing, Finanzierung, Bestandsmanagement, Projektentwicklung. 4.Auflage, Gabler: Wiesbaden, 2003.

[Econ96] Economides, N.: The economics of networks. In: International Journal of Industrial Organization 14(6). 1996. p. 673-699.

[Euro02] European Commission: ICT \& e-Business in the Real Estate Sector. In: The European e-Business Market Watch Sector Report Nr.14/2. http:/www.ebusinesswatch.org/images/stories/space/SR14-II_Real-Estate.pdf, 2002, Abruf am 12. Juli 2004.

[FoOe02] Fornefeld, M. ; Oefinger, P.: Produktkonzept zur Öffnung des Geodatenmarktes, NRW Medien GmbH. http://www.micus.de/pdf/micus produktkonzept.pdf, 2002, Abruf am 06.Oktober 2004.

$\left[\right.$ Frey $\left.^{+} 03\right]$ Frey, L. G.; Klein, H.; Koch, A.: Zeitungsverlage im Umbruch - Stimmungen und Perspektiven, Ernst \& Young. http://www.ey.com/global/download.nsf/Germany/ Studie_Zeitungsverlage_12_2003/\$file/Zeitungsverlage_12_2003.pdf, 2003, Abruf am 08. Juli 2004.

[Fuch $\left.{ }^{+} 78\right]$ Fuchs, W.; Klima, R.; Lautmann, R.; Rammstedt, O.; Wienhold, H.: Lexikon zur Soziologie. Westdeutscher Verlag: Opladen, 1978.

[Gass99] Gassmann, O.: Praxisnähe mit Fallstudienforschung. In: Wissenschaftsmanagement 6(3). 1999. p. 11-16.

[Holt04] Holtmann, C.: Organisation von Märkten - Market Engineering für den elektronischen Wertpapierhandel. Thesis, Universität Fridericiana Karlsruhe (TH), 2004.

[Irsf01] Irsfeld, N.: Internet-Marketing für die Immobilienbranche. In: Handbuch Immobilien Marketing. K.-W. Schulte; K. Hiska-Brade. Immobilien Informationsverlag: Köln, 2001, p. 393-415.

[Jenk96] Jenkis, H. W.: Kompendium der Wohnungswirtschaft. Oldenburg Verlag: München, 1996.

[KiMe02] Kittel-Wegner, E.; Meyer, J.-A.: Die Fallstudie in der betriebswirtschaftlichen Forschung und Lehre. Schriften zu Management und KMU. Universität Flensburg: 2002.

[Kunz $\left.{ }^{+} 04\right]$ Kunzelmann, M.; Weltzien, H.; Weinhardt, C.: Integration von Märkten durch neue Rollen und Services. In: E-Business - Standardisierung und Integration. F.-D. Dorloff; J. Leukel; V. Schmitz. Cuvillier Verlag: Göttingen, 2004, p. 81-99.

[Lamn89] Lamnek, S.: Qualitative Sozialforschung, Bd. 2: Methoden und Techniken. Beltz: München, Weinheim, 1989.

[Murf97] Murfeld, E.: Spezielle Betriebswirtschaftslehre der Grundstücks- und Wohnungswirtschaft. Hammonia-Verlag: Hamburg, 1997.

[Neum04] Neumann, D.: Market Engineering - A Structured Design Process for Electronic Markets. Thesis, Universität Fridericiana Karlsruhe (TH), 2004. 
[Neum ${ }^{+}$02] Neumann, D.; Holtmann, C.; Weltzien, H.; Lattemann, C.; Weinhardt, C.: Towards a Generic E-Market Design. In: The Knowledge Society: e-Commerce, eBusiness and e-Government. J. Monteiro; P. M. C. Swatman; L. V. Tavares. Kluwer Academic Publishers: Lisabon, 2002, p. 289-305.

[ObE100] Obermann, O.; Elze, R. ; Freischlad, P.: Immo Media Research 2: Die große Analyse des Immobilienmarktes im Internet. Immobilien-Informationsverlag: Köln, 2000.

[ObWi02] Obermann, O.; Wilhelm, T.: IMMO MEDIA RESEARCH 3 - Die große Analyse des Immobilienmarktes im Internet. iz IMMOBILIENZEITUNG Verlagsgesellschaft mbH: Wiesbaden, 2002.

[Per102] Perlmann, S.: Virtuelle Immobilienbörsen im gewerblichen Sektor. Materialien zur Regionalentwicklung und Raumordnung, Bd. 2. Selbstverlag / Troeger-Weiß, Gabi (Hrsg.): Kaiserslautern, 2002.

[Pico $\left.{ }^{+} 01\right]$ Picot, A.; Reichwald, R.; Wigand, R. T.: Die grenzenlose Unternehmung. Information, Organisation und Management. Gabler: Wiesbaden, 2001.

[RoBö01] Rohmert, W.; Böhm, J.: E-Business in der Immobilienwirtschaft - Neue Chancen, Märkte und Marktteilnehmer. Gabler: Wiesbaden, 2001.

[Schm93] Schmid, B.: Elektronische Märkte. In: Wirtschaftsinformatik 35(5). 1993. p. 465480.

[Schu98] Schulte, K.-W.: Immobilienökonomie - Betriebswirtschaftliche Grundlagen. Oldenburg Verlag: München, 1998.

[Smit82] Smith, V. L.: Microeconomic Systems as an Experimental Science. In: American Economic Review 72(5). 1982. p. 923-955.

[StWe03] Ströbel, M.; Weinhardt, C.: The Montreal Taxonomy for Electronic Negotiations. In: Journal of Group Decision and Negotiation 12(2). 2003. p. 143-164.

[Wein ${ }^{+}$03] Weinhardt, C.; Holtmann, C.; Neumann, D.: Market Engineering. In: Wirtschaftsinformatik 45(6). 2003. p. 635-640.

[Yin94] Yin, R. K.: Case study research : design and methods. Sage Publication: Thousand Oaks, California, 1994.

[Zerd03] Zerdick, A.: High 5, Die Zukunft der Rubrikenmärkte - Hinter den Rubriken steckt meist ein kluger Kopf!, Interview vom 10. Sept. 2003 in Berlin. http://www.immobilienscout24.de/de/download/ueberuns/interview_pdf.pdf, 2003, Abruf am 14. Juli 2004.

[Zerd $\left.{ }^{+} 01\right]$ Zerdick, A.; Picot, A.; Schrape, K.; Artope, A.; Goldhammer, K.; Heger, D. K.; Lange, U. T.; Vierkant, E.; Lopez-Escobar, E.; Silverstone, R.: Die Internet-Ölonomie. Strategien für die digitale Wirtschaft. Springer: Berlin et. al., 2001. 